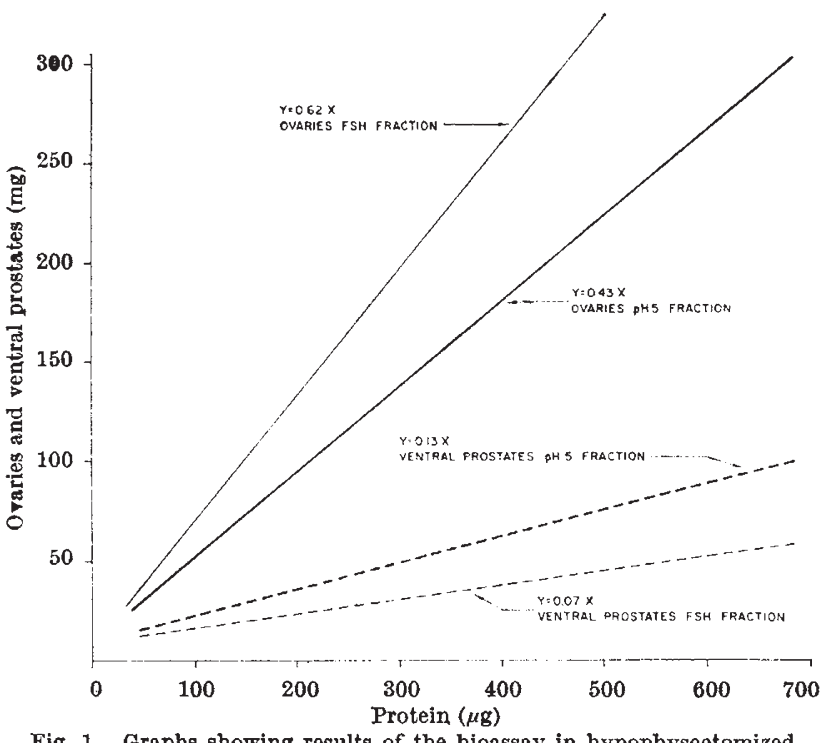

Fig. 1. Graphs showing results of the bioassay in hypophysectomized rats of the $p \mathrm{H} 5$ soluble fraction of horse pituitary glands and the FSH preparation obtained from this fraction after the third zone electro-
phoresis

this observation can be used in following the separation of two substances with overlapping biological activity.

Division of Endocrinology,

B. B. SAXENA

Department of Medicine,

Seton Hall College of Medicine,

Jersey City 4, New Jersey.

'Saxena, B. B., McShan, W. H., and Meyer, Roland K., Biochim. Biophys.

Act $0,65,394$ (1962)

2 Goldstein, A., Biostatistics, 144 (Macmillan Co., New York, 1964).

\section{Effect of Human Growth Hormone on Insulin Basic Protein Complex}

IT is generally agreed that there are two forms of insulin circulating in human blood plasma: (i) the immunologically inactive form bound to a basic $\operatorname{protein}^{1-3}$; (ii) the immunologically active free form ${ }^{4}$.

In our in vitro studies of the binding reactions between 131I-bovine insulin (Radiochemical Centre, Amersham) and a commercial insulin antibody preparation (Burroughs Wellcome) the effect of other hormones on these binding reactions was observed.

The method used to indicate 131I-insulin-antibody binding was similar to that described by Meade and

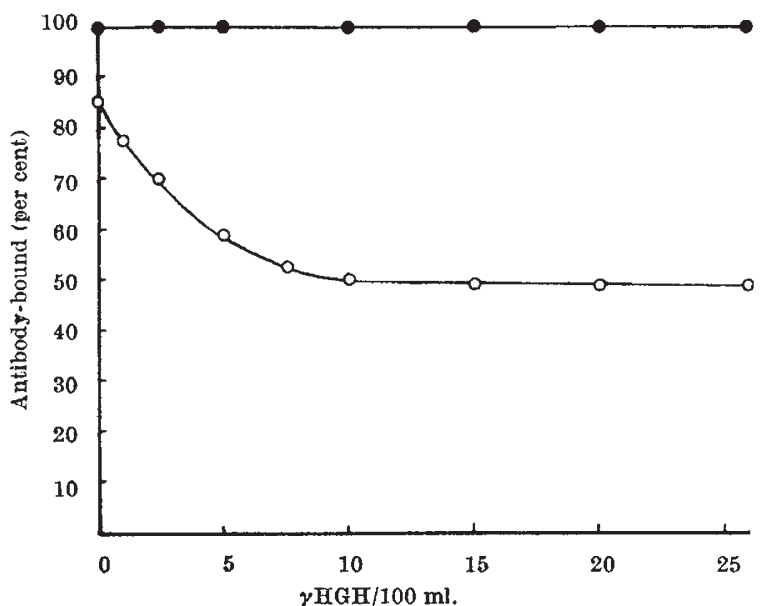

Fig. 1. Effect of added $\mathrm{HGH}$ on ${ }^{131} \mathrm{I}$-insulin-antibody binding. $\bigcirc, \mathrm{Per}$ oentage bound in serum; 0 , percentage bound in 3.95 per cent human
Kiltgaard 5,6 , who used an anion exchange resin to separate the free ${ }^{131}$ I-insulin from the insulin bound to that antibody, this giving a good index of the bound-to-free ratio of ${ }^{131}$ I-insulin.

It was found, on adding human growth hormone (HGH) (U.S. National Institutes of Health) to insulin antibody in the presence of human fasting serum, that the proportion of ${ }^{131} I$-insulin bound to antibody decreased, with increase in the amount of HGH added. When repeated with antibody in 3.95 per cent human serum albumin, no change occurred (Fig. 1).

It was postulated that $\mathrm{HGH}$ displaced endogenous insulin from the insulin-basic protein complex, and made it available for antibody binding. This theory was supported when it was found that ${ }^{131} \mathrm{I}-\mathrm{HGH}^{7}$ could be partly extracted from serum by the cation exchange resin 'Dowex 50' (Na cycle) but not from 3.95 per cent. albumin. This indicated that some HGH is bound to a basic protein in human blood plasma.

\section{J. D. Young}

Physics Department, St. Vincent's Hospital.

\section{E. P. GEORGE}

Physics Department,

University of New South Wales, Sydney, Australia.

1 Antoniades, H. N., Science, 127, 593 (1958).

${ }^{2}$ Antoniades, H. N., Endocrinology, 69, 46 (1961).

${ }^{3}$ Segal, B. M., Metabolism, 13, 8, 753 (1964).

Taylor, K. W., Disorders of Carbohydrate Metabolism, 55 (Pitman Medical Publications, London, 1962).

- Meade, R., and Kiltgaard, H., J. Nucl. Med., 3, 407 (1962).

6 George, F. P., and Young, J. D. (unpublished data).

' Hunter, W. M., and Greenwood, F. C., Nature, 194, 495 (1962).

\section{Phenolic and Indole Amines in the Urine of Schizophrenics}

THE hypothesis that schizophrenia may be caused by a metabolic abnormality of phenolic or indolic amines has gained supporting experimental results by some research groups, but conflicting results by others. This confused state was reviewed by Kety ${ }^{1}$, and the contra. dictory results could be explained by various factors related to different environmental and physical conditions which are secondary to the psychosis. An attempt was made to examine in this laboratory the excretion of various phenolic and indole amines using the subjects under careful control of environmental factors.

Seven schizophrenic patients having 5-7 year courses of the disease and eight normal subjects who were on the same diet were selected. Routine clinical examinations revealed no physical abnormalities in both groups of subjects. Medications were withdrawn for more than a month before the collection of urine and both patients and controls were engaged in the same degree of physical work. Twenty-four hour urine specimens of schizophrenics were collected under strict supervision for 3 days, but collection of a full 24-h urine failed frequently because of pathological behaviour of the patients. Thirteen specimens of complete 24-h urine could be collected. Urine was collected on the second day from normal subjects. Urine was kept in a refrigerator during the 24-h collection and then kept at $-20^{\circ} \mathrm{C}$. Quantitation of amino nitrogen, urea, creatinine, creatine and uric acid of these specimens showed that there were no pathological changes in the amounts of these nitrogen compounds in the urine of both groups.

Paper chromatograms of phenolic amines were prepared according to the method of Kakimoto and Armstrong 2 . Diazotized $p$-nitroaniline was used for the detection of phenolic amines. Paper chromatograms of indole amines were prepared in the same manner as phenolic amines and rendered visible with the $p$-dimethylaminobenzaldehyde reagent. Compounds listed in Table 1 were detected 